Research Journal of Applied Sciences, Engineering and Technology 6(14): 2681-2684, 2013

DOI:10.19026/rjaset.6.3758

ISSN: 2040-7459; e-ISSN: 2040-7467

(C) 2013 Maxwell Scientific Publication Corp.

Submitted: February 28, $2013 \quad$ Accepted: March 27, $2013 \quad$ Published: August 10, 2013

\title{
Research Article \\ Conflict-Free Automated Guided Vehicles Routing Using Multi-Objective Genetic Algorithm
}

\author{
${ }^{1}$ U.A. Umar, ${ }^{2}$ M.K.A. Ariffin, ${ }^{3}$ N. Ismail and ${ }^{4}$ S.H. Tang \\ Department of Mechanical and Manufacturing Engineering, Universiti Putra Malaysia \\ 43400 UPM Serdang, Malaysia
}

\begin{abstract}
The study presents an algorithm for conflict-free Automated Guided Vehicle (AGV) routing minimizing travel time and total job tardiness. The problem is represented using one sub-chromosome for dispatching represented with random keys and the remaining sub-chromosomes for routing represented with priority-based encoding. The algorithm used weight mapping crossover (WMX) and Insertion Mutation (IM) for priority-based representation and parameterized uniform crossover (PUX) for random-key based representation. Conflict is detected and avoided using the route occupation time of each segment. Numerical experiment was conducted on the developed algorithm.
\end{abstract}

Keywords: Automated Guided Vehicle (AGV), dispatching, multi-objective genetic algorithm, metaheuristics, optimization, routing

\section{INTRODUCTION}

In line with the immense need of inventive production strategies to reduce cost and improve overall system throughput, adoption and continues improvement of material transport system is now a reality. Automated Guided Vehicle (AGV) system provides a more flexible, cost effective and convenient means of automated material transportation system. Ineffective and inefficient $\mathrm{AGV}$ routing algorithms directly affect the overall system which may result in the total system malfunction (Le-Anh and De Koster, 2006; Vis, 2006). AGV routing problem is found to be NP complete problem (De Guzman et al., 1997). This study presents algorithms for dynamically optimizing automated guided vehicle material handling tasks and related overall system throughput using multi-objective genetic algorithm.

Routing algorithm of automated guided vehicle can be categorized as either static or dynamic. Static routing algorithm provides routing paths mapping to space domain only, while the dynamic routing algorithms provides routing path mapping in both time and space domain (Smolic-Rocak et al., 2010). With static routing algorithms the path from origin to destination is determined in prior to the request and used at all times if a material will be transported to a particular destination. Therefore, the criteria used to choose path is usually the shortest distance route. Static routing algorithm in the literature includes (Kim and Tanchoco, 1991; Krishnamurthy et al., 1993). However, static

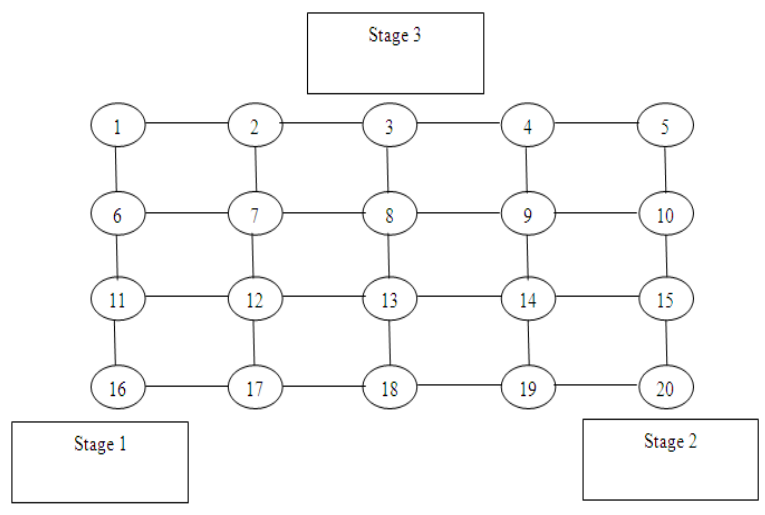

Fig. 1: Production facility layout

algorithms are not capable in considering real time traffic control functions. In dynamic routing traffic control decision utilizes the real-time information, gave rise to choice of different route usually from the particular origin to a particular destination.

Problem formulation: The manufacturing facility layout is represented by an undirected graph $\mathrm{G}=(\mathrm{N}$, A). The set of nodes $N=\left\{n_{1}, n_{2}, n_{3}, \ldots\right\}$ represent production stages and intersection of paths, while the sets of weighted $\operatorname{arcs} A=\left\{a_{1}, a_{2}, a_{3}, \ldots\right\}$ represent the routes between corresponding nodes:

$P_{v}$ represent current path, $A m$ represent the graph network adjacency matrix, $E$ represents Eligible edge set and $m$ the total jobs.

\footnotetext{
Corresponding Author: U.A. Umar, Department of Mechanical and Manufacturing Engineering, Universiti Putra Malaysia 43400 UPM Serdang, Malaysia
}

This work is licensed under a Creative Commons Attribution 4.0 International License (URL: http://creativecommons.org/licenses/by/4.0/). 
Res. J. Appl. Sci. Eng. Technol., 6(14): 2681-2684, 2013

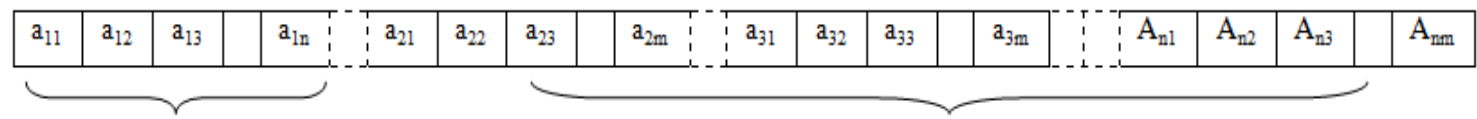

AGV Dispatching Using Random Keys-

AGV Routing Using Priority-Based Genetic Representation for m-path segment

Based Representation for $\mathrm{n}$-requests

Fig. 2: Genetic Representation Used

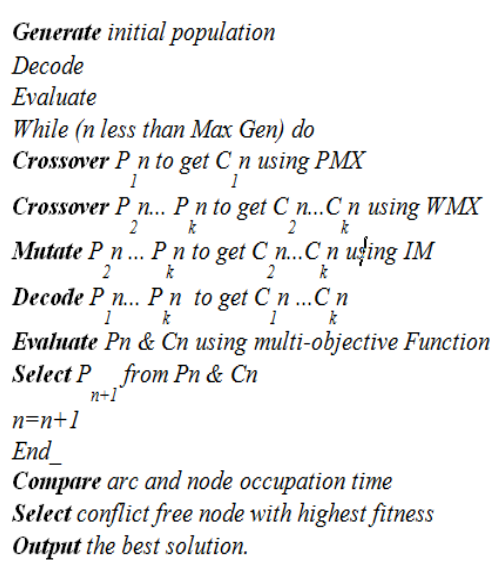

Fig. 3: Pseudocode multi-objective genetic algorithm

Let the weight $w_{i j}$ of the arc between node $i$ and $j$ represents the inverse of arc occupation time which is given by the following relation:

$$
w_{i j}=\frac{V_{c}}{d_{i j}}
$$

where, $d_{i j}$ is the length of the arc, $V_{c}$ is the speed of the AGV which is assumed to be constant throughout the transverse time.

Assumptions

The speed of the AGV is constant. Each AGV serve only one request at a specific time instance. The capacity of each buffer is sufficient to accommodate material at both production stages.

The production facility layout used for experimenting the developed algorithm as used by Nishi et al. (2011) is shown in Fig. 1.

Multi-objective genetic algorithm: Single-objective optimization is a condition where the superiority of individual solution in search space is determined by its performance toward only one objective. Other desired features can be archived by using a set of constraints before fitness evaluation or by repair process after the problem is solved. Real world problem usually need considerations of multiple objectives to get real optimum solution. In practice the objectives is often reduced to a single objective for convenience. In order to obtain better solution to a particular problem multiobjective optimization is used. Multi-criteria or multiobjective optimization utilizes two or more usually conflicting objective to evaluate the fitness of the individual solutions in the search space. Constraints can be incorporated by using equality and inequality constrains equations for decisions variables involved. Optimum solution from the all objectives involved is obtained by trade-offs between the objectives features that are usually conflicting (Deb, 2008).

Choosing a proper genetic representation for a problem is very fundamental and success determining step toward superbly solving a particular optimization problem. Inappropriate representations normally leads to no or incorrect solution to the problem at hand. Considering the nature of AGVs integrated routing which needs representing AGVs dispatching and detail path routing. Considering the fact that indiscriminate combination of nodes usually result in illegal or infeasible route and random dispatching result in overload, this study choose priority-based genetic algorithm for routing and random-key based representation for dispatching. Figure 2 shows the chromosomes representation with one sub chromosomes in random key-based representing the AGVs dispatching for $n$ request and the remaining sub chromosomes priority-based representing detail AGV route for each of the $n$ dispatched request. For more details about the representation used the reader is referred to Bean (1994), Gen et al. (1997) and Umar et al. (2012).

Let $P n$ be the parent for generation $n$ and $P_{i} n$ be the corresponding sub-chromosomes. Let $\mathrm{C} n$ be the parent for generation $n$ and $\mathrm{C}_{i} n$ is the corresponding subchromosomes. The complete pseudo code for the algorithm used is shown in Fig. 3.

Genetic operators: The algorithm used weight mapping crossover (WMX) developed by Gen et al. (1997) for sub-chromosomes represented with priority based encoding of the AGV route. Firstly, to the right segment of the locus interval random point is chosen to divide the genes into left and right segment. The two segments are interchanged between the two parent chromosomes. The offspring chromosomes right segment contains the index mapping of the sorted allele of the right segment of the other parent. The left segment of the generated offspring contains exactly the left segment the corresponding parent. Parameterized Uniform Crossover (PUX) was used for subchromosomes represented with random key based representation. In PUX, firstly the chromosome index to choose for crossover based on a randomly generated number is determined, which have same length with that of the sub-chromosomes. This randomly generated 


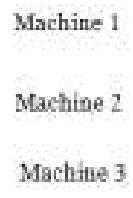

Machities 3

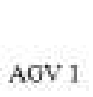

AGY 2

AGV 3
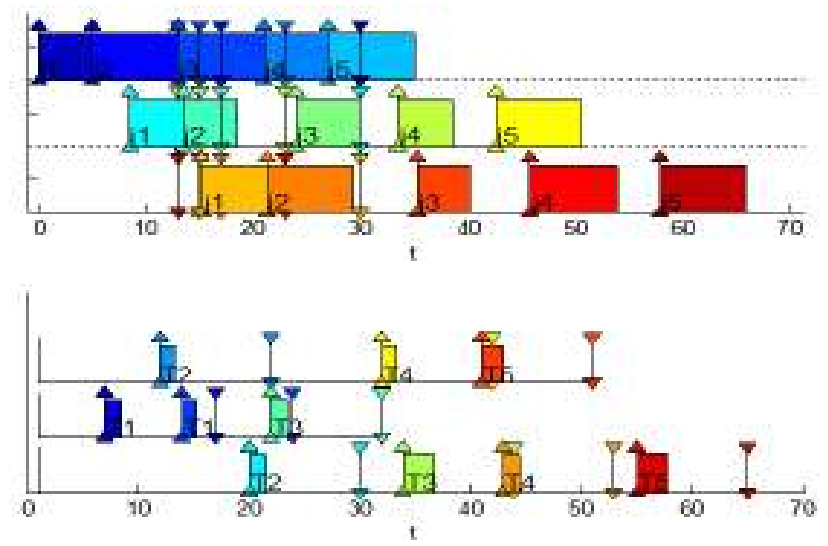

Fig. 4: Gantt chart for Jobs and AGV routing

number is the decision variable for crossover if is greater than 0.7, otherwise the chromosome is left intact without crossover for more detail about the crossover the reader is refered to Bean (1994).

The algorithm used Insertion mutation for subchromosomes represented with priority-based representation. Randomly, an index of insertion point and the insertion contain to the right were chosen. The remaining indexes to the right of the insertion point were incremented by one each. Insertion mutation was found to be great toward ensuring exploration in genetic algorithm with priority-based genetic representation (Lin and Gen, 2009).

In order choose the fittest individuals for passage to the next generation the developed algorithm uses multi-objective fitness evaluation function in Eq. (2) to evaluate the individuals in the population:

$$
f_{\text {eval }}=\min \left(m_{1} \sum_{i=1}^{n} \sum_{j=1}^{n} w_{i j} x_{i j}+m_{2} \sum_{k=1}^{m}\left(c_{k}-d_{k}\right)\right)
$$

where, $w_{i j}$ is the arc weight, $d_{k}$ is due date, $c_{k}$ is the job completion time, $m_{1}$ and $m_{2}$ are the weight of corresponding objectives. The decision variable $\mathrm{x}_{\mathrm{ij}}$ is given by the following relation:

$$
x_{i j}=\left\{\begin{array}{c}
1,(i, j) \in P v \\
0, \text { otherwise }
\end{array}\right.
$$

Route conflict that may result to deadlock and livelock is detected by using node and arc occupation times. If a conflict is detected the solution is replaced with another solution in the population.

Numerical experiment: Numerical experiment was conducted on a Pentium 4 processor (3.2-GHz), 2MB Memory and running on Window 7 Operating System. The algorithm was written in MATLAB 7.12 using population size of 20 individuals per generation crossover rate of 0.7 for both weighted mapping crossover and parameterized uniform crossover mutation rate of 0.2 . The experiment use data from Nishi et al. (2011) with three production stages and three AGVs and five jobs as shown in Fig. 1. The corresponding input data used is presented in Table 1.

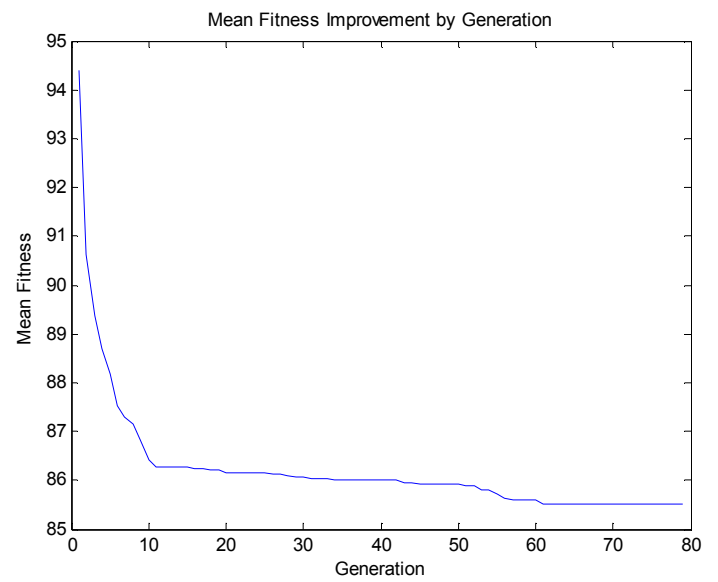

Fig. 5: Population fitness improvement history

\begin{tabular}{|c|c|c|c|c|}
\hline & \multicolumn{2}{|c|}{ Time (min) } & \multirow[b]{2}{*}{ Route } & \multirow[b]{2}{*}{ Job } \\
\hline & Start & End & & \\
\hline \multirow[t]{3}{*}{$\overline{\mathrm{AGV} 1}$} & $7: 00$ & $8: 15$ & $3-2-1-6-11-16$ & Job 2 \\
\hline & $32: 00$ & $33: 15$ & $3-2-1-6-11-16$ & Job 4 \\
\hline & 41:00 & $42: 30$ & $3-2-7-12-17-16$ & Job 5 \\
\hline \multirow[t]{3}{*}{ AGV2 } & $7: 00$ & $8: 15$ & $3-2-7-6-11-16$ & Job 1 \\
\hline & $14: 00$ & $15: 15$ & $16-17-18-19-20$ & Job 1 \\
\hline & $21: 15$ & $22: 45$ & $3-2-7-12-17-16$ & Job 3 \\
\hline \multirow[t]{4}{*}{ AGV3 } & 20:00 & $21: 15$ & $16-17-18-19-20$ & Job 2 \\
\hline & $24: 00$ & $26: 45$ & $16-11-12-7-2-3-8-9-14-15-20$ & Job 3 \\
\hline & $42: 30$ & $43: 45$ & $16-17-18-19-20$ & Job 4 \\
\hline & $55: 00$ & $57: 00$ & $3-4-9-14-13-18-17-16$ & Job 5 \\
\hline
\end{tabular}

\begin{tabular}{llllll} 
Table 1: Production data used & \multicolumn{7}{l}{} \\
\hline & Job 1 & Job 2 & Job 3 & Job 4 & Job 5 \\
\hline Weight & 30 & 17 & 15 & 7 & 3 \\
Due date & 30 & 7 & 8 & 23 & 13 \\
Processing time for stage 1 & 5 & 8 & 8 & 6 & 8 \\
Processing time for stage 2 & 5 & 5 & 6 & 5 & 8 \\
Processing time for stage 3 & 6 & 8 & 5 & 8 & 8 \\
\hline
\end{tabular}


times. If a conflict is detected the solution is replaced with another solution in the population.

The algorithm is assumed to converge whenever the selected population is not improving after 20 generation. The result obtained after about 80 generation as shown in the population history plot in Fig. 5. The results obtained is optimum compared with Nishi et al. (2011). In addition this study considers another objective of shortest time route apart from job tardiness used in the study. The detail of AGV routing is shown in Table 2. While the gantt chart for scheduling dispatching and routing is shown in Fig. 4.

\section{CONCLUSION}

The study proposed a multi-objective genetic algorithm approach to automated guided vehicle system. The optimization algorithm considers shortest time paths and job tardiness as the objectives. The problem is represented in sub-chromosomes structure in which one sub-chromosome is representing dispatching while the remaining representing automated guided vehicle routing. Due to the nature of the two representation used the genetic operators used is distinct for each representation, which in weight mapping crossover (WMX) and Insertion Mutation (IM) for priority-based representation and Parameterized Uniform Crossover (PUX) for random key-based representation. One of the main contributions of this study is the combination of two different objectives in optimizing the automated guided vehicle routing which are treated separately in the literature. The incorporation uncertainty handling into the algorithm will be the future area of research.

\section{ACKNOWLEDGMENT}

The study was supported by Research University Grant (9199811) award to Universiti Putra Malaysia.

\section{REFERENCES}

Bean, J.C., 1994. Genetic algorithms and random keys for sequencing and optimization. ORSA J. Comput., 6(2): 154-160.
De Guzman, M.C., N. Prabhu and J.M.A. Tanchoco, 1997. Complexity of the AGV shortest path and single-loop guide path layout problems. Int. J. Prod. Res., 35(8): 2083-2092.

Deb, K., 2008. Multi-Objective Optimization Using Evolutionary Algorithms. John Wiley and Sons, Chichester, New York.

Gen, M., C. Runwei and W. Dingwei, 1997. Genetic algorithms for solving shortest path problems. Proceeding of the IEEE International Conference on Evolutionary Computation. Indianapolis, IN, pp: 401-406.

Kim, C.W. and J.M.A. Tanchoco, 1991. Conflict-free shortest-time bidirectional AGV routeing. Int. J. Prod. Res., 29(12): 2377-2391.

Krishnamurthy, N.N., R. Batta and M.H. Karwan, 1993. Developing conflict-free routes for automated guided vehicles. Oper. Res., 41(6): 1077-1090.

Le-Anh, T. and M.B.M. De Koster, 2006. A review of design and control of automated guided vehicle systems. Eur. J. Oper. Res., 171(1): 1-23.

Lin, L. and M. Gen, 2009. Priority-Based Genetic Algorithm for Shortest Path Routing Problem in OSPF. In: Mitsuo, G., D. Green, O. Katai, B. McKay, A. Namatame, R. Sarker and B.T. Zhang (Eds.), Intelligent and Evolutionary Systems. Springer Berlin/Heidelberg, 187: 91-103.

Nishi, T., Y. Hiranaka and I.E.Grossmann, 2011. A bilevel decomposition algorithm for simultaneous production scheduling and conflict-free routing for automated guided vehicles. Comput. Oper. Res., 38(5): 876-888.

Smolic-Rocak, N., B. Stjepan and P. Tamara, 2010. Time windows based dynamic routing in multiAGV systems. IEEE T. Autom. Sci. Eng., 7(1): 151-155.

Umar, U.A., M.K.A. Ariffin., N. Ismail and S.H. Tang, 2012. Priority-based genetic algorithm for conflictfree automated guided vehicle routing. Proc. Eng., 50: 732-739.

Vis, I.F.A., 2006. Survey of research in the design and control of automated guided vehicle systems. Eur. J. Oper. Res., 170(3): 677-709. 